\title{
ERRATUM
}

Toshiaki Saeki • Muneo Sano • Yoshifumi Komoike Hiroshi Sonoo • Hideo Honjyo • Kazunori Ochiai

Tadashi Kobayashi $\cdot$ Kenjiro Aogi • Nobuaki Sato

Seiji Sawai · Yasuo Miyoshi • Masahiro Takeuchi

Shigemitsu Takashima

\section{No increase of breast cancer incidence in Japanese women who received hormone replacement therapy: overview of a case-control study of breast cancer risk in Japan}

Int J Clin Oncol (2008) 13:8-11

The correct name of the 11th author should be given as Yasuo Miyoshi, not Yoshio Miyoshi.

T. Saeki $(\bowtie)$

Department of Breast Oncology, Saitama Medical University School of Medicine, 1397-1 Yamane, Hidaka, Saitama 350-1241, Japan

e-mail: tsaeki@saitama-med.ac.jp

M. Sano $\cdot$ N. Sato

Niigata Cancer Center, Niigata, Japan

Y. Komoike

Osaka Medical Center for Cancer and Cardiovascular Disease,

Osaka, Japan

H. Sonoo

Kawasaki Medical School, Okayama, Japan

H. Honjyo $\cdot$ S. Sawai

Kyoto Prefectural University of Medicine, Kyoto, Japan

K. Ochiai · T. Kobayashi

Jikei University School of Medicine, Tokyo, Japan

K. Aogi $\cdot$ S. Takashima

Shikoku Cancer Center, Ehime, Japan

Y. Miyoshi

Osaka University Graduate School, Osaka, Japan

M. Takeuchi

Kitasato Medical University, Kanagama, Japan 